\title{
NORMAL REPRESENTATIONS OF BANACH JORDAN TRIPLE SYSTEMS
}

\author{
T. J. BARTON, T. DANG AND G. HORN
}

(Communicated by Paul S. Muhly)

\begin{abstract}
Some new techniques in the representation theory of Banach Jordan triple systems (i.e., $J B^{*}$-triples) are developed. These are applied to prove a Kaplansky density theorem and to show that $J B W^{*}$-triples decompose into special and exceptional parts.
\end{abstract}

In [7], L. Harris introduced a generalization of $C^{*}$-algebras which he called $J^{*}$ algebras. These are subspaces of $\mathcal{L}(H)$, the bounded operators on a Hilbert space, closed under the Jordan triple product $\{a b c\}=\left(a b^{*} c+c b^{*} a\right) / 2 . J^{*}$-algebras have subsequently come to be regarded as 'concrete' versions of Banach Jordan triple systems, or $J B^{*}$-triples. These complex Banach spaces include all $C^{*}$-algebras, $J B^{*}$-algebras, and the ranges of contractive projections on these spaces. They have a ternary algebraic structure, rather than a binary one, which is determined solely from the geometry of their unit balls. Specifically, Kaup [11, 12] has shown that each bounded symmetric domain in a complex Banach space is the biholomorphic image of the unit ball of some $J B^{*}$-triple.

The study of the representations of $J B^{*}$-triples was begun by Barton and Timoney in [1] and reached a level of maturity in Friedman and Russo [4]. The present work builds on both of those efforts. In particular, we shall prove that homomorphisms of $J B^{*}$-triples have normal extensions to their biduals, that each dual $J B^{*}$-triple (a $J B W^{*}$-triple) can be split into a direct sum of 'special' and 'exceptional' ideals, and that each $J B W^{*}$-triple has a faithful normal representation into a 'concrete' $J B W^{*}$-triple with weak*-closed range. The last two results answer questions raised by B. Russo at the 1985 GPOTS conference in the affirmative, and the last result plays a role in the characterization of continuous $J B W^{*}$-triples [10].

These results are the outcome of several informal discussions held by the authors during a visit to the University of California at Irvine by the first and third authors. We wish to express our gratitude to that institution for their hospitality.

The definition and basic properties of $J B^{*}$-triples can be found in [16]. Further background information may be found in $[1,3,4,12,14,17]$. We shall denote the triple product in a $J B^{*}$-triple $A$ by $\{x y z\}$. The Peirce spaces associated with a tripotent $e \in A$ will be denoted $A_{k}(e), k=0,1,2$, i.e., $x \in A_{k}(e) \Rightarrow\{e e x\}=k x / 2$. Without fear of confusion we shall write $A_{1}$ for the open unit ball of $A$.

A representation of a $J B^{*}$-triple $A$ in a $J B^{*}$-triple $B$ is an (algebraic) homomorphism $\varphi: A \rightarrow B$, i.e., such that $\varphi\left(\{a b c\}_{A}\right)=\{\varphi(a), \varphi(b), \varphi(c)\}_{B}$. We will show below (Lemma 1) that representations are contractive. If $A$ and $B$ are $J B W^{*}$ triples, then a representation is called normal if it is weak*-weak* continuous. The

Received by the editors December 1, 1986.

1980 Mathematics Subject Classification (1985 Revision). Primary 46H15; Secondary 47D30. 
reference to the weak* topology is not ambiguous since a $J B W^{*}$-triple has a unique predual $[\mathbf{1}, \mathbf{9}]$. Closed subtriples of $\mathcal{L}(H)$ are called $J C^{*}$-triples, and weak*-closed (ultraweakly or $\sigma$-weakly closed) subtriples of $\mathcal{L}(H)$ are called $J W^{*}$-triples. $A$ is special if it is isometrically isomorphic (=algebraically isomorphic; see Lemma 1 and $[12])$ to a $J C^{*}$-triple. $A$ is exceptional if every representation of $A$ into a $J C^{*}$-triple is zero. In [4] Friedman and Russo proved a Gelfand-Naimark Theorem: Every $J B^{*}$-triple is isometrically isomorphic to a subtriple of $\mathcal{L}(H) \oplus_{\infty} C\left(S, C_{6}\right)$, where $S$ is the Stone-Cech compactification of a discrete set and $C_{6}$ is the exceptional Car$\tan$ factor associated with the exceptional domain in $\mathbf{C}^{27}$, i.e., $C_{6}$ is the set of $3 \times 3$ hermitian matrices over the complex Cayley numbers. We shall refer to a subtriple of $\mathcal{L}(H) \oplus_{\infty} C\left(S, C_{6}\right)$ as concrete; thus, every $J B^{*}$-triple has a faithful representation as a concrete $J B^{*}$-triple. An equivalent formulation of this theorem allows the representation of a $J B^{*}$-triple to be a subtriple of $\mathcal{L}(H) \oplus_{\infty} C\left(S_{1}, C_{5}\right) \oplus_{\infty} C\left(S_{2}, C_{6}\right)$, where $S_{1}$ and $S_{2}$ are suitable topological spaces and $C_{5}$ is the exceptional Cartan factor associated with the exceptional domain in $\mathbf{C}^{16}$, i.e., $C_{5}$ is the set of $1 \times 2$ matrices over the complex Cayley numbers. Use of this form will allow us a more precise description of the exceptional part of a $J B W^{*}$-triple in Theorem 8 below.

LEMMA 1. Let $A$ and $B$ be $J B^{*}$-triples and $\varphi: A \rightarrow B$ a homomorphism. Then

(a) $\varphi$ is contractive,

(b) if $\varphi$ is injective, then $\varphi$ is an isometry.

Proof. Let $x \in A$ and let $A_{x}$ be the norm closed subtriple of $A$ generated by $x$. Then $A_{x}$ is abelian (i.e., $\{a b\{c d e\}\}=\{\{a b c\} d e\} \forall a, b, c, d, e \in A_{x}$ ), and, by continuity of the triple product in $B$, so is $C=\overline{\varphi(A)}$. Let $\Lambda_{X}$ be the set of homomorphisms of an abelian $J B^{*}$-triple $X$ into $\mathbf{C}$ endowed with the relative weak*-topology, and let $\lambda \in \Lambda_{C}$. Then $\left.\lambda \circ \varphi\right|_{A_{x}} \in \Lambda_{A_{x}}$ and so $\left\|\left.\lambda \circ \varphi\right|_{A_{x}}\right\| \leq 1$ by [12, Lemma 1.6]. By [12, Corollary 1.11] the Gelfand map isometrically embeds $C$ as a subtriple of $C_{0}\left(\Lambda_{C}\right)$, so $\|\varphi(x)\|=\sup _{\lambda \in \Lambda_{C}}|\lambda(\varphi(x))|$. Statement (a) follows.

Using the Gelfand map we may assume that $A$ and $B$ are commutative $C^{*}$ algebras and $\varphi: A \rightarrow B$ has dense image. By (a) $\varphi$ is continuous. From the separate weak*-continuity of the triple product on $A^{* *}$ and the weak*-continuity of $\varphi^{* *}: A^{* *} \rightarrow B^{* *}$ it follows that $\varphi^{* *}$ is a homomorphism with weak ${ }^{*}$-dense image. Let $e$ be the identity of the $W^{*}$-algebra $A^{* *}$ and let $u=\varphi^{* *}(e)$. Then $\varphi(A) \subseteq$ $\left(B^{* *}\right)_{2}(u) \Rightarrow B^{* *}=\left(B^{* *}\right)_{2}(u)$ and so $u$ is a unitary element in the $W^{*}$-algebra $B^{* *}$. Therefore $\psi: A^{* *} \rightarrow B^{* *}$ defined by $\psi(z)=\varphi^{* *}(z) u^{*}$ is a *algebra homomorphism. Statement (b) now follows from a well-known result of Kaplansky (cf. [15, Corollary 1.2.6]) applied to $\left.\psi\right|_{A}: A \rightarrow B^{* *}$.

We shall make frequent use of the following result, due to Horn [9].

LEMMA 2. The weak*-closed ideals of a $J B W^{*}$-triple are precisely its $M$ summands.

Proposition 3. Let $A$ and $B$ be $J B W^{*}$-triples and $\varphi: A \rightarrow B$ a normal representation. Then $\varphi(A)$ is weak*-closed in $B$.

Proof. By Lemma $2 \operatorname{ker} \varphi$ is an $M$-summand in $A$. Thus, there is an $M$ projection $P$ on $A$ such that $\varphi P(A)=\varphi(A)$ and $\varphi P$ is injective, and so is an isometric isomorphism by Lemma 1 . Thus, the closed unit ball of $\varphi(A)$ is $\varphi\left(\overline{A_{1}}\right)$ which is weak*-compact in $B$ by Alaoglu's Theorem and the weak*-continuity of $\varphi$. 
Hence, the Krein-Smullyan Theorem implies that $\varphi(A)$ is a weak*-closed subspace of $B$.

Our principal tool in this article is the following theorem which establishes the normal extension of a representation into a $J B W^{*}$-triple.

THEOREM 4. Let $A$ be a J $B^{*}$-triple, $B$ a JBW*-triple, and $\varphi: A \rightarrow B a$ representation. Then there is a unique normal extension of $\varphi$ to a representation $\tilde{\varphi}: A^{* *} \rightarrow B$.

ProOF. Let $\iota: B_{*} \rightarrow B^{*}$ be the canonical inclusion, where $B_{*}$ is the predual of $B$, and define $T=\iota^{*}$. Then $T$ is weak*-weak* continuous and it readily follows that $T(x)=x \forall x \in B$. By [2] $B$ is a subtriple of $B^{* *}$ and so $\left.T\right|_{B}$ is a homomorphism. Since $B$ is weak*-dense in $B^{* *}$ and $\{\cdot, \cdot, \cdot\}_{B^{* *}}$ is separately weak* continuous [1], $T$ is a homomorphism on all of $B^{* *}$.

Define $\tilde{\varphi}=T \varphi^{* *}$. Then $\tilde{\varphi}$ is weak*-weak* continuous. As in the above paragraph $\varphi^{* *}: A^{* *} \rightarrow B^{* *}$ is a homomorphism and thus so is $\tilde{\varphi}$. For $x \in A, T \varphi^{* *}(x)=$ $T \varphi(x)=\varphi(x)$ so $\tilde{\varphi}$ extends $\varphi$. Any other normal extension of $\tilde{\varphi}$ must agree with $\tilde{\varphi}$ on the weak*-dense subset $A$ and so coincides with $\tilde{\varphi}$.

Throughout the rest of this paper we shall place a tilde above a representation to indicate its normal extension.

COROLlaRY 5 (KAPLANSKY Density ThEOREM). Let $A$ be a weak*-dense norm closed subtriple of a $J B W^{*}$-triple $B$. Then $A_{1}$ is weak*-dense in $B_{1}$.

Proof. Let $\iota: A \rightarrow B$ be the inclusion map. By Proposition $3 \tilde{\iota}\left(A^{* *}\right)$ is weak*closed in $B$. The hypothesis then entails $\tilde{\imath}\left(A^{* *}\right)=B$, and as the weak*-closure of $A_{1}$ in $A^{* *}$ is ${\overline{\left(A^{* *}\right)}}_{1}$ we have $\tilde{\imath}\left({\overline{A_{1}}}^{\text {weak }^{*}}\right)=\overline{B_{1}}$. But

$$
\tilde{\imath}\left({\overline{A_{1}}}^{\text {weak }^{*}}\right) \subseteq{\overline{\tilde{\iota}\left(A_{1}\right)}}^{\text {weak }^{*}}={\overline{\iota\left(A_{1}\right)}}^{\text {weak }^{*}}={\overline{A_{1}}}^{\text {weak }^{*}} .
$$

For the next result it will be convenient to distinguish between a Banach space and its canonical image in its bidual. Let $A$ be a $J B^{*}$-triple and let $j: A \rightarrow A^{* *}$ be the canonical inclusion. Each weak ${ }^{*}$-closed ideal $M$ in $A^{* *}$ is an $M$-summand by Lemma 2 . With the $M$-projection $P: A^{* *} \rightarrow M$ we define the canonical representation of $A$ corresponding to $M$ to be the map $x \rightarrow P j(x)$. That this defines a representation follows from Lemma 2. Two representations $\varphi_{k}: A \rightarrow B_{k}, k=1,2$, are equivalent if there is an algebraic isomorphism $\psi: B_{1} \rightarrow B_{2}$ such that $\varphi_{2}=\psi \varphi_{1}$.

Proposition 6. Let $A$ be a $J B^{*}$-triple, $B$ a JBW*-triple, and $\varphi: A \rightarrow B$ a representation with weak*-dense image. Then $B$ is algebraically isomorphic to a weak ${ }^{*}$-closed ideal $M$ in $A^{* *}$ and $\varphi$ is equivalent to the canonical representation of $A$ corresponding to $M$.

ProOF. Since $\operatorname{ker} \tilde{\varphi}$ is a weak*-closed ideal in $A^{* *}$, by Lemma 2 there is a weak*closed ideal $M$ in $A^{* *}$ with $A^{* *}=M \oplus_{\infty} \operatorname{ker} \tilde{\varphi}$. Let $x \rightarrow P j(x)$ be the canonical representation of $A$ corresponding to $M$ and define $\psi=\left.\varphi\right|_{M}: M \rightarrow B$. Then $\psi$ is a normal monomorphism onto a weak*-dense subset of $B$. By Proposition 3 we have in fact $\psi(M)=B$, i.e., $\psi$ is an algebraic isomorphism of $M$ onto $B$. Finally, for $x \in A, \varphi(x)=\tilde{\varphi}(j(x))=\tilde{\varphi} P j(x)=\psi P j(x)$.

By removing the weak*-dense hypothesis in Proposition 6 and replacing $B$ with the weak*-closure of $\varphi(A)$ in the above proof we obtain the following. 
COROLlaRY 7. Let $\varphi: A \rightarrow B$ be a representation of a $J B^{*}$-triple into a $J B W^{*}$-triple. Then the weak*-closure of $\varphi(A)$ is algebraically isomorphic to a weak ${ }^{*}$-closed ideal in $A^{* *}$. Moreover, $\overline{\varphi(A)}{ }^{\text {weak }^{*}}=\tilde{\varphi}\left(A^{* *}\right)$.

THEOREM 8. Every $J B W^{*}$-triple $A$ has a unique decomposition $A=A_{\mathrm{sp}} \oplus_{\infty}$ $A_{\mathrm{ex}}$, where $A_{\mathrm{sp}}$ is isometrically isomorphic to a $J C^{*}$-triple and $A_{\mathrm{ex}}$ is isometrically isomorphic to $C\left(S_{1}, C_{5}\right) \oplus_{\infty} C\left(S_{2}, C_{6}\right)$ for some hyperstonean spaces $S_{1}$ and $S_{2}$. $A_{\mathrm{ex}}$ coincides with the purely exceptional ideal of [4, Theorem 2].

Proof. By $[9,(4.13)] A$ has a decomposition $A=V \oplus_{\infty} W$, where $V$ is a type I $J B W^{*}$-triple and $W$ is a $J B W^{*}$-triple with no nonzero abelian tripotents. $V$ decomposes as desired by the classification theorem for type I $J B W^{*}$-triples [8]. Thus, it suffices to prove that $W$ is isometrically isomorphic to a $J C^{*}$-triple.

By [13] the maximal tripotents (i.e., those tripotents $e \in W$ such that $W_{0}(e)=0$ ) in a $J B^{*}$-triple coincide with the real and the complex extreme points of the closed unit ball. Since $W$ is a dual space, we may choose a maximal tripotent $e \in W$ by the Krein-Milman Theorem. By [9] $W_{2}(e)$ is a $J B W^{*}$-algebra. It follows that $W_{2}(e)$ contains no nonzero abelian projections. Hence, $W_{2}(e)$ contains four strongly connected, pairwise orthogonal projections $e_{1}, e_{2}, e_{3}, e_{4}$ with $e_{1}+e_{2}+e_{3}+e_{4}=e$ (see $[6,5.2 .15]$ ), i.e., there are $x_{i j} \in W_{1}\left(e_{i}\right) \cap W_{1}\left(e_{j}\right), i, j=1,2,3,4$, such that

$$
\left\{x_{i j}, e_{i}, x_{i j}\right\}=e_{j} \text {. }
$$

Let $\varphi: W \rightarrow C$ be a representation into an exceptional Cartan factor $C$ (so $C=C_{5}$ or $\left.C_{6}\right)$. Suppose $\exists x \in W$ with $\varphi(x) \neq 0$. By maximality of $e \exists y \in W$ with $x=\{e e y\}$ and so $0 \neq\{\varphi(e), \varphi(e), \varphi(y)\} \Rightarrow \varphi(e) \neq 0$. By $(*) \varphi\left(e_{i}\right), i=1,2,3,4$, are either all zero or all nonzero, and since $e_{1}+e_{2}+e_{3}+e_{4}=e$ they must all be nonzero. Consequently, $C$ has rank at least 4 , a contradiction since $C_{5}$ has rank 2 and $C_{6}$ has rank 3. Thus, $\varphi=0$ and so $W$ is isometrically isomorphic to a $J C^{*}$-triple by [4, Corollary 4].

If $A=\tilde{A}_{\mathrm{sp}} \oplus_{\infty} \tilde{A}_{\mathrm{ex}}$ is another decomposition of $A$ into special and exceptional parts, then $A_{\text {ex }}=\left(A_{\text {ex }} \cap \tilde{A}_{\text {ex }}\right) \oplus\left(A_{\text {ex }} \cap \tilde{A}_{\text {sp }}\right)$ by [9, (4.2)]. But $A_{\text {ex }} \cap \tilde{A}_{\text {sp }}$ is both special and exceptional and so is zero. Thus, $A_{\text {ex }} \subseteq \tilde{A}_{\text {ex }}$, and by symmetry $A_{\text {ex }}=\tilde{A}_{\text {ex }}$ and $A_{\text {sp }}=\tilde{A}_{\text {sp }}$.

To prove the second assertion of the theorem we must show that every representation of $A_{\text {ex }}$ into a $J C^{*}$-triple is zero. Let $\varphi: C\left(S_{1}, C_{5}\right) \oplus_{\infty} C\left(S_{2}, C_{6}\right) \rightarrow B$ be such a representation and assume $\varphi \neq 0$. Consider $C_{5}$ embedded in $C\left(S_{1}, C_{5}\right)$ as the subspace of constant functions. The tripotent $f(s) \equiv\left[\begin{array}{ll}1 & 0\end{array}\right]$ is maximal in $C\left(S_{1}, C_{5}\right)$ and so $\varphi\left(C_{5}\right) \neq 0$. Since $C_{5}$ has no proper ideal, $\left.\varphi\right|_{C_{5}}$ is faithful, which contradicts the exceptionality of $C_{5}$. A similar argument for $C_{6}$ yields $\varphi=0$.

COROLLARY 9. Every $J B W^{*}$-triple has a faithful normal representation onto a weak*-closed $J B W^{*}$-subtriple of $\mathcal{L}(H) \oplus_{\infty} C\left(S_{1}, C_{5}\right) \oplus_{\infty} C\left(S_{2}, C_{6}\right)$ for some hyperstonean spaces $S_{1}$ and $S_{2}$. In particular, a special $J B W^{*}$-triple has a faithful normal representation as a $\mathrm{JW}^{*}$-triple.

Proof. Let $A$ be a $J B W^{*}$-triple and $A=A_{\mathrm{sp}} \oplus_{\infty} A_{\mathrm{ex}}$ the decomposition given by Theorem 6. $A_{\mathrm{ex}}$ is isometrically isomorphic to $C\left(S_{1}, C_{5}\right) \oplus_{\infty} C\left(S_{2}, C_{6}\right)$, and the normality of this representation follows from the uniqueness of preduals of $J B W^{*}$-triples (see [5, Theorem 12]). Thus, we may assume that $A$ is special.

Let $B \subset \mathcal{L}(H)$ be a $J C^{*}$-triple and let $\varphi: B \rightarrow A$ be an algebraic isomorphism. By Proposition 6, $A$ is algebraically isomorphic to a weak*-closed ideal in $B^{* *}$. 
Algebraic isomorphisms are surjective isometries by Lemma 1 and therefore are weak*-weak* continuous by uniqueness of preduals, so it will suffice to show that $B^{* *}$ is algebraically isomorphic to a $J W^{*}$-triple.

Let $\iota: B \rightarrow \mathcal{L}(H)$ be the inclusion map. Then $\iota^{* *}: B^{* *} \rightarrow \mathcal{L}(H)^{* *}$ is an isometry and so $\iota^{* *}$ is a weak*-weak* continuous monomorphism. By $[6,1.1 .23] \iota^{* *}\left(A^{* *}\right)$ is weak*-closed in $\mathcal{L}(H)^{* *}$. Let $\psi: \mathcal{L}(H)^{* *} \rightarrow \mathcal{L}(K)$ be a faithful normal representation of $\mathcal{L}(H)^{* *}$ as a von Neumann algebra, where $K$ is some complex Hilbert space (cf. [15, Theorem 1.16.7]). Then $\psi \circ \iota^{* *}$ is an algebraic isomorphism of $B^{* *}$ onto a weak*-closed subtriple of $\mathcal{L}(K)$, i.e., onto a $J W^{*}$-triple.

\section{REFERENCES}

1. T. Barton and R. Timoney, Weak*-continuity of Jordan triple products and applications, Math. Scand. 59 (1986), 177-191.

2. S. Dineen, Complete holomorphic vector fields on the second dual of a Banach space, Math. Scand. 59 (1986), 131-142.

3. Y. Friedman and B. Russo, Structure of the predual of a $J B W^{*}$-triple, J. Reine Angew. Math. 356 (1985), 67-89.

4. __ The Gelfand-Naimark Theorem for JB*-triples, Duke Math. J. 53 (1986), 139-148.

5. G. Godefroy, Espaces de Banach: Existence et unicité de certains prédaux, Ann. Inst. Fourier (Grenoble) 28 (1978), 87-105.

6. H. Hanche-Olsen and E. Størmer, Jordan operator algebras, Pitman, London, 1984.

7. L. Harris, $A$ generalization of $C^{*}$-algebras, Proc. London Math. Soc. 42 (1981), 331-361.

8. G. Horn, Klassifikation der JBW*-Tripel vom Typ I, Dissertation, Tübingen, December 1984.

9. _ Characterization of the predual and ideal structure of a JBW*-triple, Math. Scand. (to appear).

10. G. Horn and E. Neher, Classification of continuous $J B W^{*}$-triples, Trans. Amer. Math. Soc. (to appear).

11. W. Kaup, Algebraic characterization of symmetric complex Banach manifolds, Math. Ann. 228 (1977), 39-64.

12. __ A Riemann mapping theorem for bounded symmetric domains in complex Banach spaces, Math. Z. 183 (1983), 503-529.

13. W. Kaup and H. Upmeier, Jordan algebras and symmetric Siegel domains in Banach spaces, Math. Z. 157 (1977), 179-200.

14. M. Koecher, An elementary approach to bounded symmetric domains, Lecture Notes, Rice University, 1969.

15. S. Sakai, $C^{*}$-algebras and $W^{*}$-algebras, Springer-Verlag, Berlin, Heidelberg and New York, 1971.

16. H. Upmeier, Symmetric Banach manifolds and Jordan $C^{*}$-algebras, North-Holland, Amsterdam, 1985.

17. J. P. Vigué, Le groupe des automorphismes analytiques d'un domaine borné d'un espace de Banach complexe. Application aux domains bornes symmétriques, Ann. Sci. École Norm. Sup. (4) 9 (1976), 203-282.

Department of Mathematics, Memphis State University, Memphis, Tennessee 38152 (Current address of T. J. Barton)

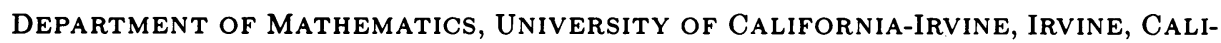
FORNIA 92717 (Current address of T. Dang)

Mathematisches Institut, Universität Tübingen, D-7400 Tübingen, West GERMANY

Current address (G. Horn): Siemens AG, Corporate Research and Technology, Otto-HahnRing 6, D-8000 Munich 83, West Germany 